\title{
Violência entre parceiros íntimos, oferta de leite materno, substitutos e uso de mamadeiras no primeiro ano de vida
}

\author{
Intimate partner violence, breastfeeding, breastmilk substitutes \\ and baby bottle use in the first year of life
}

Raquel de Souza Mezzavilla (https://orcid.org/0000-0002-6323-4336) ${ }^{1}$

Gabriela Vasconcellos de Barros Vianna (https://orcid.org/0000-0002-5409-5856) ${ }^{2}$

Ana Cristina Lindsay (https://orcid.org/0000-0002-2520-0493) ${ }^{3}$

Maria Helena Hasselmann (https://orcid.org/0000-0002-3106-1522) ${ }^{2}$

${ }^{1}$ Departamento ou Programa de Pós-

Graduação, Universidade

do Estado do Rio de Janeiro.

Rua São Francisco Xavier

524 bloco D $12^{\circ}$ andar sala

12024 Maracanã. 20559-900

Rio de Janeiro RJ Brasil.

raquelmezzavilla@

hotmail.com

${ }^{2}$ Departamento de Nutrição

Social, Instituto de Nutrição,

Universidade do Estado

do Rio de Janeiro. Rio de

Janeiro RJ Brasil.

${ }^{3}$ Department of Exercise

and Health Sciences, College

of Nursing and Health

Sciences, University of

Massachusetts Boston EUA.

\begin{abstract}
This article aims to investigate the relationship between intimate partner physical violence (IPPV) and breastfeeding (BF), use of breastmilk substitutes (BMS) and bottle-feeding among children aged 12 to 15 months. This is a cross-sectional study with mothers in primary care facilities of the city of Rio de Janeiro. IPPV was identified by the Brazilian version of the Conflict Tactics Scales 1-Form $R$ and feeding practices were identified by a 24-hour Dietary Recall. Associations were verified by logistic regression with odds ratio (OR) estimates and $95 \%$ confidence intervals. BF was offered to $58.5 \%$ of the children and BMS to $88.5 \%$. Also, $70.5 \%$ of the children used a baby bottle. Physical violence was observed in $26.7 \%$ of couples. Households where couples physically abuse each other are more likely to not breastfeed, to use breastmilk substitutes and bottle-feed, when compared to households without physical violence. The results highlight the need to investigate intrafamily relationships in cases where inadequate breastfeeding practices are identified, and to enable health professionals to support families in conflict situations.
\end{abstract}

Key words Breastfeeding, Domestic violence, Bottle-feeding
Resumo O objetivo deste artigo é investigar a relação da violência física entre parceiros intimos (VFPI) e a oferta de leite materno (LM), os substitutos do leite materno (SLM) e o uso de mamadeiras entre crianças entre 12 e 15 meses. Estudo transversal com mães em unidades de saúde do município do Rio de Janeiro, realizado entre junho de 2005 e dezembro de 2009. A VFPI foi identificada pela versão brasileira da Conflict Tactics Scales 1- Form $R$ e as práticas de alimentação foram identificadas por recordatório de 24 horas. As associações foram verificadas por regressão logistica mediante estimativas de razão de chances $(R C)$ e intervalos de confiança de $95 \%$. O leite materno foi ofertado a $58,5 \%$ das crianças e os substitutos do leite materno a $88,5 \%$, e 70,5\% das crianças usavam mamadeira. A violência física foi observada em $26,7 \%$ dos casais. Em lares onde os casais se agridem fisicamente há uma maior chance de não ofertar leite materno, maior chance de ofertar substitutos do leite materno e de usar mamadeira comparado aos lares onde não existe violência física. Os resultados chamam atenção para a necessidade de se investigar as relações intrafamiliares em casos que são identificadas práticas inadequadas de aleitamento, e de capacitar os profissionais de saúde para apoiar as famílias em situações de conflito.

Palavras-chave Aleitamento materno, Violência doméstica, Alimentação artificial 


\section{Introdução}

Comprovadamente o aleitamento materno é a forma ideal de alimentação para o crescimento e desenvolvimento infantil, recomendado exclusivamente nos primeiros seis meses de vida e complementado por alimentos saudáveis até os dois anos de idade ou mais ${ }^{1}$. No entanto, dados de pesquisas realizadas em todo país revelam que no primeiro mês de vida $18 \%$ das crianças consumiram água, chás e outros leites e, antes dos seis meses de idade, consumiram comida salgada e frutas. Do mesmo modo, a introdução tardia de alimentos também é observada ${ }^{2,5}$.

Ao longo dos anos, diversos estudos têm investigado as consequências de práticas alimentares inadequadas à saúde das crianças durante os primeiros mil dias de vida ${ }^{6-8}$. A desnutrição, por exemplo, ligada às principais causas de morte do mundo, está associada diretamente à ingestão inadequada de alimentos, principalmente o abandono precoce do aleitamento materno exclusivo e a alimentação complementar inadequa$\mathrm{da}^{9-10}$. Paralelamente, nas últimas décadas, a literatura tem destacado a importância do aumento do sobrepeso e da obesidade na infância ${ }^{12-14}$. Segundo dados globais, em 2016, quase 40,6 milhões de crianças menores de cinco anos estavam acima do peso ou obesas ${ }^{15}$.

Práticas apropriadas de aleitamento materno e alimentação complementar desempenham um papel significativo na melhoria da saúde e da nutrição das crianças. Alguns autores estimam que a alimentação complementar adequada e oportuna, juntamente com a amamentação contínua por até dois anos de idade, poderiam salvar vidas de milhões de crianças menores de cinco anos de idade por ano ${ }^{16,17}$. Além disso, são responsáveis por conferir benefícios expressivos a longo prazo durante adolescência e idade adulta ${ }^{7}$.

Acrescenta-se que, utensílios como bicos, mamadeiras e chuquinhas são fontes de contaminação, portanto, inseguros para a criança e prejudiciais ao desenvolvimento da fala, da mastigação e da aceitação do peito ${ }^{18-20}$.

Alguns fatores relacionados às práticas inadequadas de aleitamento materno destacam-se: menor idade materna; baixa escolaridade; experiência anterior negativa em relação à amamentação; desconhecimento sobre o manejo da amamentação e suas vantagens, e consequências negativas do uso de mamadeiras, de bicos, de chupetas e de fórmulas infantis; o retorno ao trabalho no pós-parto imediato; e ainda, a separação entre mãe e filho nos primeiros meses de vida; a falta de apoio familiar e a presença de depressão e violência familiar ${ }^{2,4,21-27}$.

Pesquisas acerca da relação da violência entre parceiros íntimos (VPI) e as práticas de aleitamento revelam que existe uma associação entre a violência e a interrupção do aleitamento materno exclusivo nos primeiros meses de vida da criança e, mais recentemente, a introdução de líquidos e sólidos antes do quarto mês de vida ${ }^{28,29}$.

Resultados de uma recente revisão sistemática da literatura apontam para uma menor chance de amamentar e de iniciar o aleitamento materno e maior chance de interromper o aleitamento materno exclusivo entre as mulheres que são vítimas da violência. Além disso, a VPI está associada à introdução de alimentos artificiais, a uma alimentação mista e à oferta de líquidos e de alimentos sólidos precocemente ${ }^{30}$. Ressalta-se ainda que a faixa etária das investigações encontradas limita-se aos seis meses de vida.

O papel do ambiente familiar no crescimento e alimentação das crianças tem sido investigado em pesquisas recentes ${ }^{27,29,30}$, no entanto, a relação da violência entre parceiros íntimos e as práticas de aleitamento em crianças no primeiro ano de vida ainda merece ser mais explorada.

Nessa perspectiva, o objetivo central do presente estudo foi investigar a associação da violência física entre parceiros íntimos e a oferta de leite materno, os substitutos do leite materno e o uso de mamadeira em crianças no primeiro ano de vida atendidas em Unidades Básicas de Saúde (UBS) do município do Rio de Janeiro.

\section{Método}

\section{Desenho e população de estudo}

O presente estudo é uma investigação do tipo seccional cujas informações são originárias de um estudo de coorte prospectivo que acompanhou, entre junho de 2005 e dezembro de 2009 , o crescimento durante o primeiro ano de vida de recém-nascidos acolhidos em quatro Unidades Básicas de Saúde do município do Rio de Janeiro. As entrevistas com as mães ocorreram em seis momentos: primeiro, segundo, terceiro, quinto, sétimo e $13^{\circ}$ meses de vida da criança.

Com o intuito de aumentar a adesão, somente as crianças que participaram das duas primeiras entrevistas compuseram a linha de base da coorte e foram acompanhadas pelos meses subsequentes. Também com o mesmo objetivo, o acompanhamento coincidiu com as ocasiões em que as 
mães retornavam para consulta com o pediatra e/ou vacinação da criança, conforme calendário de vacinas do Ministério da Saúde.

\section{Critérios de inclusão}

A amostra foi composta por 217 mães e crianças que participaram das entrevistas da linha de base e do $13^{\circ}$ mês de vida de seus filhos, e que apresentaram informações sobre o desfecho (oferta de alimentos nas últimas 24 horas antecedentes à entrevista) e a exposição de interesse (violência entre parceiros íntimos).

\section{Critérios de exclusão}

Foram excluídos gemelares, crianças com mães adotivas e com idade superior a 15 meses.

\section{Mensuração e coleta de dados}

As informações utilizadas neste estudo foram coletadas por meio de entrevistas com questionários pré-testados, realizadas por pesquisadores (nutricionistas) previamente treinados.

\section{Mensuração do desfecho: práticas de aleitamento}

As práticas de aleitamento nas 24 horas antecedentes à entrevista foram investigadas na entrevista do $13^{\circ}$ mês de vida, através de um instrumento com perguntas fechadas, adaptado dos utilizados nos inquéritos em dias de campanha nacionais de multivacinação, e que incluiu questões sobre o consumo de: leite materno, outros tipos de leite e outros alimentos, o que inclui água, chás e outros líquidos.

As variáveis leite materno (LM) e substitutos do leite materno (SLM), este último constituído por fórmulas infantis e leite de vaca, foram categorizadas de forma dicotômica: recebeu $=0$, não recebeu $=1$. Além dessas variáveis, foi investigado o uso de mamadeira, também categorizado de forma dicotômica.

\section{Mensuração da exposição central: violência física entre parceiros íntimos}

Para a obtenção de dados referentes à violência entre parceiros íntimos, as mães foram questionadas a respeito de estratégias utilizadas por elas e seus parceiros para resolverem possíveis desavenças. $\mathrm{O}$ instrumento utilizado para a coleta dessas informações foi a versão em português da
Conflict Tactics Scales - Form R (CTS-1) ${ }^{31}$, aplicada no $12^{\circ}$ mês de vida da criança.

O instrumento CTS- 1 contém 18 itens e se propõe a mensurar as estratégias utilizadas para resolver possíveis desavenças ocorridas doze meses anteriores à entrevista e, indiretamente, captar uma situação de violência. O formulário abrange três táticas para lidar com conflitos: "argumentação" (uso de discussão racional - itens a-c); "violência verbal" (uso verbal e não verbal com a finalidade de ameaçar, ferir ou magoar itens d-f, h-j); e "violência física" (uso de força física para lidar com conflitos - itens $\mathrm{k}-\mathrm{s}$ ). A violência física tem duas subescalas: violência física menor (itens $\mathrm{k}-\mathrm{m}$ ) e violência física grave (itens $\mathrm{n}$-s). Para este estudo foram utilizadas as escalas de violência física.

Para cada item da escala, são possíveis quatro opções de respostas: "Algumas vezes reagiu assim"; "Muitas vezes reagiu desse modo"; "Já reagiu assim, mas não nos últimos 12 meses"; "Nunca reagiu dessa forma" 32 .

Os relacionamentos apreciados foram o do parceiro em relação à mulher na condição de mãe e o trato recíproco. A violência física entre parceiros íntimos (VFPI) foi classificada como positiva quando as mulheres responderam (1) ou (2) a pelo menos um dos itens da escala de violência física (itens k-s), seja como vítima ou perpetradora. Essa classificação também foi utilizada para as violências físicas menores (itens $\mathrm{k}-\mathrm{m}$ ) e as graves (itens $n-s)$. As categorias de violência física global, física menor e física grave foram dicotomizadas como proposto por Straus ${ }^{33}$.

\section{Mensuração das covariáveis}

As covariáveis foram investigadas na linha de base do estudo e consideraram posse de utensílios domésticos, uso de rede social, escolaridade materna, idade materna, transtornos mentais comuns e trabalho atual materno.

A variável "posse de utensílios domésticos" foi avaliada a partir dos seguintes itens pertinentes na residência: aparelho de som, máquina de lavar, secadora de roupa, ferro a vapor, forno elétrico, freezer, multiprocessador, aspirador de pó, forno de microondas, computador e aparelho de DVD. Ao levar em conta a mediana da distribuição, o ponto de corte $>3$ foi considerado maior posse de utensílios domésticos.

Para mensurar a rede social, utilizou-se questões referentes à quantidade de amigos e parentes com quem a mulher pôde contar nos últimos 12 meses $^{34}$. Esta variável foi analisada de forma dicotômica (0-2 e três ou mais parentes e/ou amigos). 
Os transtornos mentais comuns (TMC) foram avaliados por meio de um instrumento com 12 itens, o General Health Questionnaire (GHQ$12)^{35}$, com 12 itens que refletem sentimentos de depressão, ansiedade, falta de confiança e incapacidade de lidar com situações habituais. Cada item apresentava quatro opções de resposta, em que as duas primeiras classificadas como ausência do sintoma (0 ponto atribuído) e as duas últimas como presença do sintoma (1 ponto atribuído). Após o somatório, obtivemos uma escala de 0 a 12 e, a partir dessa escala, a variável de transtornos mentais foi classificada em três categorias: transtornos mentais comuns ( $\geq 3$ respostas positivas), graves ( $\geq 5$ respostas positivas), e sintomas depressivos ( $\geq 9$ respostas positivas) 36 .

\section{Processamento e análise dos dados}

Os questionários foram checados, codificados e $100 \%$ das digitações revisadas. Pequeno percentual de erro encontrado foi corrigido. Utilizou-se o programa Epiinfo $6.04^{37}$ para o armazenamento dos dados e as análises foram feitas no pacote estatístico $R$-Project versão $2.11 .1 .^{38} \mathrm{e}$ no Stata $11^{39}$.

Inicialmente, foram estimadas prevalências da variável dependente, da exposição central e das covariáveis. As relações entre as covariáveis e as práticas de aleitamento foram verificadas mediante estimativas de razão de chances (Odds Ratio-OR) e respectivos intervalos de confiança de 95\% (IC95\%).

Para as análises multivariadas foi realizado modelo de regressão logística ajustado pelas covariáveis citadas na literatura como relevantes para essa relação: posse de utensílios domésticos, trabalho materno atual, idade materna, rede social e TMC (suspeição de depressão) ${ }^{4,25,26,40}$. Considera-se poder de $80 \%$ para detectar como significante $(\mathrm{p}<0,05)$ uma razão de chances de 1.6 , considerando prevalência de não oferta de leite materno entre não expostos de $38 \%$.

\section{Questões éticas}

O projeto foi aprovado pelo Comitê de Ética em Pesquisa do Instituto de Medicina Social da Universidade do Estado do Rio de Janeiro.

Os autores declaram não haver conflitos de interesse.

\section{Resultados}

Das 217 crianças, 50,9\% eram do sexo masculino e tinham idade média no momento da entrevista de 12,8 meses $\pm 1,08$. As prevalências de consumo de leite materno e de seus substitutos foram, respectivamente, $58,5 \%$ e $88,5 \%$, além disso, chama atenção que mais de $75 \%$ das crianças faziam uso de mamadeiras. Entre parceiros íntimos, as prevalências de violência física global, menor e grave foram, respectivamente, $26,7 \%, 25,8 \%$ e $11,1 \%$ (Tabela 1).

Nas análises bivariadas foram observadas associações significativas entre a violência física global e a não oferta de leite materno, bem como à oferta de substitutos do leite materno e ao uso de mamadeira (Tabela 2).

Nas análises multivariadas, mesmo após ajuste pelas covariáveis posse de utensílios domésticos, trabalho atual materno, idade materna, rede social e suspeição de depressão, as associações permaneceram significativas. Casais que se agridem fisicamente têm 2,14 vezes mais chances de não ofertar o leite materno (IC 1,06 - 4,31); 5,15 vezes mais chances de ofertar substitutos do leite materno (IC 1,13 - 23,4); e 2,71 vezes mais chances de fazer uso de mamadeira (IC 1.19 - 6.16), comparados aos casais que não se agridem fisicamente (Tabela 2).

\section{Discussão}

O presente estudo é pioneiro em avaliar a relação da VFPI e as práticas de aleitamento de crianças no primeiro ano de vida. Os resultados mostram que os casais que se agridem fisicamente apresentam duas vezes mais chances de não ofertarem leite materno, cinco vezes mais chances de ofertarem substitutos do leite materno e duas vezes mais chances de usarem mamadeira para alimentar seus filhos, comparados aos casais que não se agridem.

Em geral, estudos anteriores que investigaram a violência entre parceiros íntimos como um fator de risco para práticas alimentares inadequadas de crianças focalizaram o aleitamento materno nos primeiros meses de vida, e consideraram como fatores a menor intenção de amamentar ${ }^{41}$, a interrupção precoce do aleitamento materno exclusivo $^{28,29,42}$, a menor chance de iniciar o aleitamento materno ${ }^{42,43}$ e a menor duração do aleitamento materno ${ }^{44}$.

Yount et $\mathrm{al}^{45}$, em uma revisão da literatura sobre as consequências da violência doméstica 
Tabela 1. Características gerais da população de quatro UBS do município do Rio de Janeiro, 20052009.

\begin{tabular}{|c|c|c|c|}
\hline Características & $\mathbf{n}$ & $(\%)$ & Total \\
\hline \multicolumn{4}{|l|}{ Posse de utensílios domésticos } \\
\hline Três ou mais & 129 & 59,4 & 215 \\
\hline Menos que três & 86 & 39,6 & \\
\hline \multicolumn{4}{|l|}{ Escolaridade materna } \\
\hline Fundamental completo ou & 136 & 68,7 & 198 \\
\hline mais & 62 & 31,3 & \\
\hline \multicolumn{4}{|l|}{ Fundamental incompleto } \\
\hline \multicolumn{4}{|l|}{ Trabalho materno atual } \\
\hline Sim & 74 & 34,3 & 216 \\
\hline Não & 142 & 65,4 & \\
\hline \multicolumn{4}{|l|}{ Idade Materna } \\
\hline$<20$ & 30 & 15,1 & \\
\hline $20-34$ & 146 & 73,4 & 199 \\
\hline$\geq 35$ & 23 & 11,6 & \\
\hline \multicolumn{4}{|l|}{ Suspeição de depressão } \\
\hline Sim & 11 & 94,9 & 214 \\
\hline Não & 203 & 5,1 & \\
\hline \multicolumn{4}{|l|}{ Rede de apoio } \\
\hline Nenhum & 8 & 4,0 & \\
\hline 1 & 31 & 15,7 & 198 \\
\hline 2 & 25 & 12,6 & \\
\hline 3 ou mais & 134 & 67,7 & \\
\hline Leite materno & & & 217 \\
\hline $\operatorname{Sim}$ & 127 & 58,5 & \\
\hline Não & 90 & 41,5 & \\
\hline Substitutos do leite materno & & & 217 \\
\hline $\operatorname{Sim}$ & 192 & 88,5 & \\
\hline Não & 25 & 11,7 & \\
\hline Mamadeira & & & 217 \\
\hline Sim & 153 & 70,5 & \\
\hline Não & 64 & 29,5 & \\
\hline \multicolumn{4}{|l|}{ VFPI Global } \\
\hline Sim & 58 & 26,7 & 217 \\
\hline Não & 159 & 73,3 & \\
\hline \multicolumn{4}{|l|}{ VFPI Menor } \\
\hline Sim & 56 & 25,8 & 217 \\
\hline Não & 161 & 74,2 & \\
\hline \multicolumn{4}{|l|}{ VFPI Grave } \\
\hline $\operatorname{Sim}$ & 24 & 11,1 & 217 \\
\hline Não & 193 & 88,9 & \\
\hline
\end{tabular}

Fonte: Elaborada pelas autoras.

no crescimento de crianças, observaram que a saúde física, nutricional e mental materna foram intermediárias desse efeito. Os autores ressaltaram que a partir de comportamentos de riscos, tais como os cuidados inadequados no pré-natal e com a criança, levou-se a desfechos desfavoráveis ao estado nutricional infantil.

Além disso, outros autores sugerem que as mulheres maltratadas podem apresentar baixa auto-estima, falta de autonomia e deficiências físicas e mentais para desenvolver as atividades do seu dia a dia, como a escolha e preparação de alimentos, e acabam por oferecer leite de fórmulas a seus filhos. Autores ainda pontuam que a depressão e a ansiedade materna podem resultar em apoio social e cuidados com o aleitamento materno reduzidos ${ }^{46-51}$. Deste modo, é possível apontar a saúde mental da mulher como uma variável interveniente da relação entre VFPI e as práticas de aleitamento subótimas.

No que diz respeito à alimentação com substitutos do leite materno, além dos riscos relacionados à criança e à mãe, alimentar com leite artificial aumenta o consumo de recursos escassos e a acumulação de lixo não-biodegradável, repercute no orçamento familiar, além de alteração da relação do vínculo entre a mãe e o bebê $\hat{e}^{52-54}$.

Ainda que a recomendação atual dos órgãos competentes é que as crianças entre 6 meses e 2 anos de idade devam receber a comida da família e leite materno ${ }^{3,52,53}$. Segundo a OMS, menos de uma em cada cinco crianças são amamentados por 12 meses em países de alta renda e apenas duas crianças em cada três crianças entre 6 meses e 2 anos de idade recebem leite materno em países de baixa e média renda ${ }^{54}$.

O leite materno é o alimento ideal para crianças na primeira infância por ser fonte de nutrientes, embora sozinho não possa suprir as necessidades de nutrientes e energia a partir do sexto mês de vida. Além disso, o aleitamento materno continuado traz benefícios para ambas, mãe e criança, como por exemplo, acarreta menor risco de câncer de mama nas mulheres e a menor chance de obesidade na infância ${ }^{55,56}$.

No que diz respeito à violência entre parceiros, na população estudada, $26,7 \%$ dos casais se agrediam fisicamente e cerca de $11 \%$ se agrediam gravemente. Ao longo dos anos as pesquisas sobre a magnitude da violência entre parceiros íntimos têm se intensificado, como por exemplo, um estudo transversal de base populacional na zona urbana do município de Florianópolis revelou a prevalência de violência física de qualquer tipo (17\%), violência física moderada $(16,6 \%)$ e grave (7,3\%) entre homens e mulheres. Os autores observaram que quanto mais grave o ato de violência, maior a ocorrência de mulheres agredidas ${ }^{57}$. Acrescenta-se que a maioria das vítimas de agressão física é submetida a vários atos de violência durante longos períodos ${ }^{58,59}$.

Vale mencionar que as populações pesquisadas são diversas, e que os referidos estudos investigaram diferentes tipos de violências e utiliza- 
Tabela 2, Razões de chances (brutas e ajustadas), intervalos de confiança de 95\% (IC 95\%) e nível de significância (p), entre as variáveis de exposição e os desfechos, Rio de Janeiro, 2005-2009,

\begin{tabular}{lrrrr}
\hline & \multicolumn{3}{c}{ Violência física entre parceiros íntimos (global) } \\
\cline { 2 - 5 } Desfechos & \multicolumn{3}{c}{ Brutas } & \multicolumn{2}{c}{ Ajustadas* } \\
\cline { 2 - 5 } & OR & $\begin{array}{c}\text { IC 95\% } \\
\text { valor de p }\end{array}$ & OR & $\begin{array}{c}\text { IC 95\% } \\
\text { valor de } \mathbf{~ p ~}\end{array}$ \\
\cline { 2 - 5 } Não oferta de leite materno & 1 & $1,013,74$ & 2,14 & $1,064,31$ \\
Oferta de leite artificial & 1,94 & 0,031 & & 0,03 \\
Uso de mamadeira & 4,71 & $1,142,6$ & 5,15 & $1,1323,4$ \\
& 1 & 0,02 & & 0,033 \\
& 1,86 & $0,884,19$ & 2,71 & $1,196,16$ \\
& 1 & 0,08 & & 0,01 \\
\hline
\end{tabular}

*Análises ajustadas pelas covariáveis: posse de utensílios domésticos, trabalho atual materno, idade materna, rede social e suspeição de depressão.

Fonte: Elaborada pelas autoras.

ram diferentes instrumentos nas suas aferições, o que dificultou a comparação dos seus achados. Apesar dessas divergências, a dimensão do fenômeno é alta e esforços merecem ser feitos a fim de melhorar esta situação, construir estratégias que incentivem o diálogo e propiciar o exercício da cultura da paz, o que parece ser um caminho nesta direção ${ }^{60}$.

Cabe mencionar algumas limitações desse estudo. Apesar de se tratar de um delineamento transversal, foi possível identificar que o tempo de exposição à violência, 12 meses anteriores à entrevista, apresentou associação com as práticas de aleitamento, observadas a partir do recordatório de 24 horas garantindo uma temporalidade de causa e efeito.

A aferição dos desfechos também merece comentários. Nesta mensuração utilizou-se um recordatório de 24 horas, o qual apresenta alimentos predefinidos e opções de respostas fechadas. Segundo Carvalhaes ${ }^{61}$, a utilização dos dados atuais sobre alimentação infantil minimiza erros relacionados à memória do informante. Além disso, a pouca variedade da alimentação das crianças menores de um ano também torna o recordatório de 24 horas um bom instrumento para avaliar o consumo alimentar desse grupo etário, principalmente quando apresenta a relação dos principais alimentos consumidos pelos lactentes, o que leva à redução de erros na coleta de dados. A vantagem de utilização desse método é a menor probabilidade de mudança do hábito alimentar, pois a informação é coletada depois do consumo ${ }^{62}$.

Além disso, destaca-se o fato de o presente estudo utilizar uma escala para medir a VFPI adaptada para a população brasileira e cujas propriedades psicométricas apresentam-se adequa- das, fundamental para o sucesso destas ações de investigação da violência.

À luz dessas considerações, os achados da presente investigação apontam para a relevância da VFPI na determinação das práticas de aleitamento de crianças entre 12 e 15 meses de vida. Mesmo que diversos fatores já tenham sido apontados na literatura como determinantes dessas práticas, até o momento nenhum outro estudo havia investigado essa relação com a VFPI, nessa faixa etária e em contextos nacionais.

A identificação da violência entre parceiros íntimos é imprescindível para a implementação de estratégias de apoio à vítima e esses achados contribuem para a elaboração de ações de promoção e de prevenção, tanto em relação às práticas alimentares de crianças nos primeiros anos de vida quanto à violência entre parceiros íntimos. É importante que essas estratégias incorporem a violência na família como mais um fator relacionado à inadequada introdução de alimentos, à interrupção do aleitamento materno continuado, às escolhas alimentares da família e, consequentemente, como mais um alvo de intervenção.

Destaca-se ainda que a violência é um fenômeno complexo que envolve não só um indivíduo, mas todos os integrantes do núcleo familiar ${ }^{3}$, 63, 64. As unidades de saúde são locais estratégicos de ação, uma vez que as mulheres e crianças, em geral, são os que mais buscam os serviços de saúde em decorrência de danos físicos, mentais ou emocionais. Acrescenta-se que o contexto complexo que envolve a vítima e a sua relação com o agressor faz com que o enfrentamento da violência extrapole os serviços de saúde, o que envolve os diferentes setores como segurança pública, justiça e trabalho, bem como toda sociedade civil 
organizada, para configurar redes integradas de atendimento.

\section{Conclusão}

Este estudo foi pioneiro ao investigar a relação da VFPI e as práticas de aleitamento de crianças no primeiro ano de vida. A violência física entre casais está associada à não oferta do aleitamento materno e ao uso de mamadeiras. A violência apresenta consequências não só para a vítima, mas para todos os integrantes do núcleo familiar, especialmente às mulheres, principais responsáveis pelo cuidado dos seus filhos e das práticas alimentares da família.

Um olhar mais atento do profissional de saúde aos aspectos relacionados a estes fenômenos poderá auxiliar nas decisões das vítimas de violência sobre a forma de lidar com a situação de conflito, estimular sua autonomia e melhorar sua relação com as práticas alimentares.

\section{Colaboradores}

RS Mezzavilla e MH Hasselmann foram responsáveis pela concepção e desenho do estudo, realizaram as análises e interpretação dos dados, elaboraram, revisaram e aprovaram a versão final do manuscrito. GVB Vianna e AC Lindsay elaboraram, revisaram e aprovaram a versão final do manuscrito.

\section{Financiamento}

Fundação Carlos Chagas Filho de Amparo à Pesquisa do Estado do Rio de Janeiro (FAPERJ), Coordenação de Aperfeiçoamento do Pessoal de Nível Superior (CAPES) e Conselho Nacional de Desenvolvimento Científico e Tecnológico (CNPq). 


\section{Referências}

1. Victora CG, Bahl R, Barros AJD, França GVA, Horton S, Krasevec J, Murch S, Sankar MJ, Walker N, Rollins NC. Breastfeeding in the 21st century: epidemiology, mechanisms, and lifelong effect. The Lancet 2016; 387 (10017):475-490.

2. Brasil. Ministério da Saúde (MS). Pesquisa nacional de demografia e saúde da criança e da mulher. Secretaria de Ciência, Tecnologia e Insumos Estratégicos Departamento de Ciência e Tecnologia. Brasília: MS, 2008.

3. Brasil. Ministério da Saúde (MS). Saúde da criança: nutrição infantil. Aleitamento materno e alimentação complementar. Séria A, normas e manuais técnicos. Caderno de Atenção Básica. n.23. Brasília: DF; 2009.

4. Coelho LC, Asakura L, Sachs A, Erbert I, Novaes CRL, Gimeno SGA. Sistema de Vigilância Alimentar e Nutricional/SISVAN: conhecendo as práticas alimentares de crianças menores de 24 meses. Cien Saude Colet 2015; 20(3):727-738.

5. Andrade HS, Pessoa RA, Donizete LCV. Fatores relacionados ao desmame precoce do aleitamento materno. Rev Bras Med Fam Comunidade 2018; 13(40):1-11.

6. Monteiro PO, Victora CG. Rapid growth in infancy and childhood and obesity in later life-A systematic review. Obesity Review 2005; 6:143-154.

7. Black RE, Victora CG, Walker SP, Bhutta ZA, Christian P, Onis Mercedes, Ezzati M, Grantham-McGregor S, Katz J, Martorell R, Uauy R, Maternal and Child Nutrition Study Group. Maternal and child undernutrition and overweight in low-income and middle-income countries. Lancet 2013; 382(9890):427-451.

8. Stirnemann J, Villar J, Salomon LJ, Ohuma E, Ruyan P, Altman DG, Nosten F, Craik R, Munim S, Cheikh Ismail L, Barros FC, Lambert A, Norris S, Carvalho M, Jaffer YA, Noble JA, Bertino E, Gravett MG, Purwar M, Victora CG, Uauy R, Bhutta Z, Kennedy S, Papageorghiou AT. International estimated fetal weight standards of the INTERGROWTH-21st Project. Ultrasound Obstet Gynecol 2017; 49: 478-486.

9. Victoria CG, Onis M, Hallal PC, Blössner M, Shirimpton R. Worldwide timing of growth faltering: revisiting implications for interventions. Pediatrics 2010, 125(3): 473-480.

10. World Health Organization (WHO). Essential Nutrition Actions: Improving maternal, newborn, infant and young child health and nutrition. Geneva: WHO; 2013.

11. Matanda DJ, Mittelmark MB, Kigaru DM. Breast-, complementary and bottle-feeding practices in Kenya: stagnant trends were experienced from 1998 to 2009. Nutr Res 2014; 34(6):507-517.

12. World Health Organization (WHO). Infant and young child feeding: model chapter for textbooks for medical students and allied health professionals. Geneva: WHO; 2009.

13. Emandi AC, Puiu M, Gafencu M, Pienar C. Overweight and obesity in school age children in western Romania. Rev Med Chir Soc Med Nat Iasi 2013; 117(1):36-45.

14. Hunsberger M. Early feeding practices and family structure: associations with overweight in children. Proc Nutr Soc 2014;(73):132-136.
15. Fernandes G, Sridhar D. World Bank and the Global Financing Facility. BMJ 2017; 358:j3395.

16. Jones AD, Ickes SB, Smith LE, Mbuya MN, Chasekwa B, Heidkamp RA, Menon P, Zongrone AA, Stoltzfus RJ. World Health Organization infant and young child feeding indicators and their associations with child anthropometry: a synthesis of recent findings. Matern Child Nutr 2014; 10:1-17.

17. Cordero MJA, Lopez AMS, Banos NM, Villar NM, Ruiz ME, Rodríguez EH. Lactancia materna como prevención del sobrepeso y la obesidad en el niño y el adolescente; revisión sistemática. Nutr Hosp 2015; 31(2):606-620.

18. Marques NM, Lira PIC, Lima MC, Silva NL, Batista Filho M, Huttly SRA, Ashworth A. Breastfeeding and early weaning practices in Northeast Brazil: a longitudinal study. Pediatrics 2001; 108(4):e66.

19. Soares MEM, Giugliani ERJ, Braun ML, Salgado ACN, Oliveira AP, Aguiar PR. Uso de chupeta e sua relação com o desmame precoce em população de crianças nascidas em Hospital Amigo da Criança. J Pediatr (Rio J) 2003; 79(4):309-316.

20. Brasil. Rede Internacional em Defesa do Direito de Amamentar (IBFAN). Alimentos para crianças de até 3 anos, bicos, chupetas e mamadeiras. $1^{\text {a }}$ ed. Jundiaí: IBFAN; 2007.

21. Almeida JAG. Amamentação: um híbrido naturezacultura. J Pediatr 2004; 80(Supl.5):119-125.

22. Brunken GS, Silva SM, Venâncio SI. Fatores associados à interrupção precoce do aleitamento materno exclusivo e à introdução tardia da alimentação complementar no centro-oeste brasileiro. J Pediatr 2006; 82(6):445-451.

23. Lindsay AC, Machado MT, Sussner KM, Hardwick CK, Peterson and Karen E. Infant-feeding practices and beliefs about complementary feeding among low -income Brazilian mothers: a qualitative study. Food Nutr Bull 2008; 29(1):15-24.

24. Silva LMP, Venâncio SI, Marchioni DML. Práticas de alimentação complementar no primeiro ano de vida e fatores associados, Rev Nutr 2010; 23(6):983-992.

25. Wasser H, Bentley M, Borja J, Davis Goldman B, Thompson A, Slining M, Adair L. Infants Perceived as "Fussy" Are More Likely to Receive Complementary Foods Before 4 Months. Pediatrics 2011; 127(2):229237.

26. Kronborg H, Foverskov E, Vaeth M. Predictors for early introduction of solid food among Danish mothers and infants: an observational study. BMC Pediatrics 2014, 14:243.

27. Pivetta HMF, BrazMM, Pozzebon NM, Freire AB, Real AA, Cocco VM, Sperandio FF. Prevalência de aleitamento materno e fatores associados: uma revisão de literature. Rev Cienc Med Biol 2018; 17(1):95-101.

28. Moraes CL, Oliveira ASD, Reichenheim ME, Lobato G. Severe physical violence between intimate partners during pregnancy: a risk factor for early cessation of exclusive breast-feeding. Public Health Nutr 2011; 14(12):2148-2155. 
29. Zureick-Brown S, Lavilla K, Yount KM. Intimate partner violence and infant feeding practices in India: a cross-sectional study. Matern Child Nutr 2013; 11(4):792-802.

30. Mezzavilla RS, Ferreira MF, Curioni CC, Lindsay AC, Hasselmann MH. Violência entre parceiros íntimos e práticas de aleitamento materno: uma revisão sistemática de estudos observacionais. J Pediatr 2018, 94(3):226-237.

31. Hasselmann MH, Reichenheim ME. Adaptação transcultural da versão em português da "Conflict Tactics Scales FormR" (CTS-1) usada para aferir violência no casal: Equivalências semântica e de mensuração. Cad Saude Publica 2003; 19(4):1083-1093.

32. Straus MA, Gelles JR. Physical violence in American families: risk factors and adoptions to violence in $8.145 f a-$ milies. New Brunswick: NJ: Transaction Publishers; 1995.

33. Straus MA. Measuring Intrafamily Conflict and Violence: The Conflict Tactics (CT) Scales J Marriage Fam 1979; 41(1):75-88.

34. Chor D, Griep RH, Lopes CS, Faerstein E. Medidas de rede e apoio social no Estudo Pró-Saúde: pré-testes e estudo piloto. Cad Saude Publica 2001; 17(4):887-896.

35. Goldberg D, Williams P. A user's guide to the general health questionnaire. [about 126 p.] UK: nfer-Nelson; 1988.

36. Hassan BK, Werneck GL, Hasselmann MH. Saúde mental materna e estado nutricional de crianças aos seis meses de vida. Rev Saude Publica 2016, 50(7):1-9.

37. Dean AG, Dean JA, Burton AH, Dicker RC, Coulombier D. EpiInfo, Version 6.04: a word processing, database, and statistics program for epidemiology on microcomputers. Atlanta, Georgia, USA: Centers for Disease Control; 1995.

38. R Development Core Team. $R$ : A language and environment for statistical computing. Vienna: R Foundation for Statistical Computing; 1993.

39. StataCorp. Stata Statistical Software: Release 12. College Station, TX: StataCorp LP; 2011.

40. Barchi F, Winter SC, Dougherty D, Ramaphane P, Solomon PL. The association of depressive symptoms and intimate partner violence against women in Northwestern Botswana. J Interper Violence 2018 [online]; s.v.(s.n.)

41. Sipsma HL, Divney AA, Magriples U, Hansen N, Gordon D, Kershaw T. Breastfeeding intentions among pregnant adolescents and young adults and their partners. Breastfeed Med 2013; 8(4):374-380.

42. Misch ES, Yount KM. Intimate partner violence and breastfeeding in Africa. Matern Child Health J 2014; 18(3):688-697.

43. Lau Y, Chan KS. Influence of intimate partner violence during pregnancy and early postpartum depressive symptoms on breastfeeding among chinese women in Hong Kong. J Midwifery Womens Health 2007; 52(2):15-20.

44. Sipsma HL, Magriples U, Divney A, Gordon D, Gabzdyl E, Kershaw T. Breastfeeding behavior among adolescents: initiation, duration, and exclusivity. J Adolesc Health 2013; 53(3):394-400.
45. Yount KMA, Digirolamo AMB, Ramakrishnan U. Impacts of domestic violence on child growth and nutrition: a conceptual review of the pathways of influence. Soc Sci Med 2011, 72(9):1534-1554.

46. Silverman JG, Decker MR, Reed E, Raj A. Intimate partner violence victimization prior to and during pregnancy among women residing in 26 U.S. states: Associations with maternal and neonatal health. $A m J$ Obstet Gynecol 2006; 195(1):140-148.

47. McLearn KT, Minkovitz CS, Strobino DM, Marks E, Hou W. Maternal Depressive Symptoms at 2 to 4 Months Post Partum and Early Parenting Practices. Arch Pediatr Adolesc 2006; 160(3):279-284.

48. Fried LE, Cabral H, Amaro H, Aschengrau A. Lifetime and During Pregnancy Experience of Violence and the Risk of Low Birth Weight and Preterm Birth. JMidwifery Womens Health 2008; 53(6):522-528.

49. Muller RT, Goebel-Fabbri AE, Diamond T, Dinklage D. Social support and the relationship between family and community violence exposure and psychopathology among high risk adolescents. Child Abuse Negl 2000; 24(4):449-464.

50. Rodriguez E, Lasch KE, Chandra P, Lee J. The relation of family violence, employment status welfare benefits and alcohol drinking in the United States. West $J$ Med 2001; 174(5):317-323.

51. Surkan PJ, Ryan LM; Vieira LM; Berkman LF; Peterson KE. Maternal social and psychological conditions and physical growth in low-income children in Piauí, Northeast Brazil. Soc Sci Med 2007; 64(2):375-388.

52. World Health Organization (WHO). Infant and young child feeding. Geneva: WHO; 2014.

53. Brasil. Ministério da Saúde (MS). ENPACS : Estratégia Nacional Para Alimentação Complementar Saudável: Caderno do Tutor / Ministério da Saúde, Rede Internacional em Defesa do Direito de Amamentar (IBFAN). Brasília: MS; 2010.

54. World Health Organization (WHO). Report of the commission on ending childhood obesity. Geneva: WHO; 2016.

55. Horodynski MA, Stommel M.Nutrition education aimed at toddlers: an intervention study. Pediatr Nurs 2005; 31(5):364, 367-372.

56. American Academy of Pediatrics (AAP).Complementary feeding. In: Kleiman RE, Ed.Pediatric Nutrition Handbook. $6^{\mathrm{a}}$ ed. Elk Grove Village, IL: American Academy of Pediatrics; 2009.

57. Lindner SR, Coelho EBS, Bolsoni CC, Rojas PF, BoingAF. Prevalência de violência física por parceiro íntimo em homens e mulheres de Florianópolis, Santa Catarina, Brasil: estudo de base Populacional. Cad Saude Publica 2015; 31(4):815-826.

58. Gartland D, Hemphill SA, Hegarty K, Brown SJ. Intimate Partner Violence During Pregnancy and the First Year Postpartum in an Australian Pregnancy Cohort Study. Matern Child Health J 2010; 15(5):570578.

59. Mohammad HE, Sahraean L, Bahrami T. Domestic abusebefore, during and after pregnancy in Jahrom, Islamic Republic of Iran. East Mediterr Health J 2010, 16(7):752-758. 
60. Wathen CN, MacGregor JCD, Hammerton J, Coben JH, Herrman H, Stewart DE, MacMillan HL, Rede de Pesquisa PreVAiL. Priorities for research in child maltreatment, intimate partner violence and resilience to violence exposures: results of an international Delphi consensus development process. BMC Public Health 2012; 12:684

61. Carvalhaes MABL, Parada CMGL, Manoel CM, Venâncio SY. Diagnóstico da situação do aleitamento materno em área urbana do Sudeste do Brasil: utilização de metodologia simplificada. Rev Saude Publica 1998; 32(5):430-436.

62. Buzzard M. 24-hours dietary recall and food record methods. In: Willett WC. Nutritional Epidemiology. $2^{\mathrm{a}}$ ed. Oxford: Oxford University Press; 1998.

63. Assis SG, Avanci JQ, Pesce RP, Ximenes LF. Situação de crianças e adolescentes brasileiros em relação à saúde mental e à violência. Cien Saude Colet 2009; 14(2):349-361.

64. Brasil. Ministério da Saúde (MS). Secretaria de Atenção à Saúde. Departamento de Atenção Básica. Por uma cultura da paz, a promoção da saúde e a prevenção da violência / Ministério da Saúde, Secretaria de Atenção à Saúde, Departamento de Atenção Básica. Brasília: MS; 2009b.

Artigo apresentado em 08/04/2019

Aprovado em 24/07/2019

Versão final apresentada em 26/07/2019

Editores-chefes: Romeu Gomes, Antônio Augusto Moura da Silva 